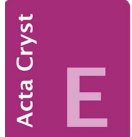
COMMUNICATIONS

ISSN 2056-9890

\section{Crystal structure of 1-\{1-[2-(phenylselan- yl)phenyl]-1H-1,2,3-triazol-4-yl\}cyclo- hexan-1-ol}

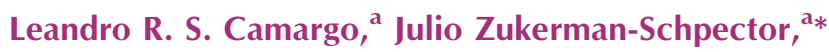
Anna M. Deobald, ${ }^{b} \neq$ Antonio L. Braga ${ }^{c}$ and Edward R. T. Tiekink $^{\mathrm{d}}$

a Departmento de Química, Universidade Federal de São Carlos, 13565-905 São Carlos, SP, Brazil, 'b Departmento de Química, Universidade Federal de Santa Maria, 97105-900 Santa Maria, RS, Brazil, `Departmento de Química, Universidade Federal de Santa Catarina, 88040-900 Florianópolis, SC, Brazil, and department of Chemistry, University of Malaya, 50603 Kuala Lumpur, Malaysia. *Correspondence e-mail: julio@power.ufscar.br

Received 11 February 2015; accepted 16 February 2015

Edited by P. C. Healy, Griffith University, Australia

Two independent molecules, $A$ and $B$, comprise the asymmetric unit of the title compound, $\mathrm{C}_{20} \mathrm{H}_{21} \mathrm{~N}_{3} \mathrm{OSe}$. While the benzene ring directly bound to the central triazole ring is inclined to the same extent in both molecules [dihedral angles $=40.41$ (12) (molecule $A$ ) and $\left.44.14(12)^{\circ}(B)\right]$, greater differences are apparent in the dihedral angles between the Se-bound rings, i.e. 74.28 (12) (molecule $A$ ) and $89.91(11)^{\circ}$ $(B)$. Close intramolecular Se $\cdots \mathrm{N}$ interactions of 2.9311 (18) (molecule $A$ ) and 2.9482 (18) $\AA(B)$ are noted. In the crystal, supramolecular chains along the $a$ axis are formed via $\mathrm{O}-$ $\mathrm{H} \cdots \mathrm{N}$ hydrogen bonding. These are connected into layers via $\mathrm{C}-\mathrm{H} \cdots \mathrm{O}$ and $\mathrm{C}-\mathrm{H} \cdots \mathrm{N}$ interactions; these stack along $(01 \overline{1})$ without directional intermolecular interactions between them.

Keywords: crystal structure; organoselenium; hydrogen bonding; Se...N halogen bonding.

CCDC reference: 1049508

\section{Related literature}

For background and synthesis of arylseleno-1,2,3-triazoles, including of the title compound, see: Deobald et al. (2011). For Se...N interactions, see: Pati \& Zade (2014).

¥ Present address: Instituto Federal de Educação, Ciência e Tecnologia Farroupilha Rua Erechim, 860 - Bairro Planalto, 98280-000 Panambi, RS, Brazil.

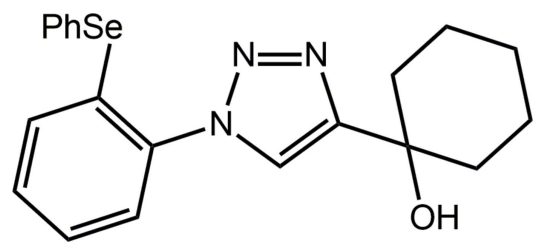

\section{Experimental}

\subsection{Crystal data}

$\mathrm{C}_{20} \mathrm{H}_{21} \mathrm{~N}_{3} \mathrm{OSe}$

$M_{r}=398.36$

Triclinic, $P \overline{1}$

$a=10.7480(4) \AA$

$b=13.7497(6) \AA$

$c=13.8849(5) \AA$

$\alpha=112.432(4)^{\circ}$

$\beta=92.889(3)^{\circ}$

$$
\begin{aligned}
& \gamma=104.059(3)^{\circ} \\
& V=1816.28(13) \AA^{3} \\
& Z=4 \\
& \mathrm{Cu} K \alpha \text { radiation } \\
& \mu=2.89 \mathrm{~mm}^{-1} \\
& T=100 \mathrm{~K} \\
& 0.20 \times 0.10 \times 0.05 \mathrm{~mm}
\end{aligned}
$$

\subsection{Data collection}

Agilent SuperNova CCD diffractometer

Absorption correction: multi-scan (CrysAlis PRO; Agilent, 2011)

$T_{\min }=0.884, T_{\max }=1.000$

26101 measured reflections 7291 independent reflections 7014 reflections with $I>2 \sigma(I)$ $R_{\text {int }}=0.087$

\subsection{Refinement}

$R\left[F^{2}>2 \sigma\left(F^{2}\right)\right]=0.039$

$w R\left(F^{2}\right)=0.107$

$S=1.08$

7291 reflections
453 parameters

$\mathrm{H}$-atom parameters constrained

$\Delta \rho_{\max }=1.06 \mathrm{e}^{-3}$

$\Delta \rho_{\min }=-1.11 \mathrm{e}^{-3}$
Table 1

Hydrogen-bond geometry $\left(\AA{ }^{\circ}\right)$.

\begin{tabular}{lllll}
\hline$D-\mathrm{H} \cdots A$ & $D-\mathrm{H}$ & $\mathrm{H} \cdots A$ & $D \cdots A$ & $D-\mathrm{H} \cdots A$ \\
\hline $\mathrm{C} 8-\mathrm{H} 8 \cdots \mathrm{N} 5$ & 0.95 & 2.32 & $3.229(3)$ & 159 \\
$\mathrm{C} 18-\mathrm{H} 18 \cdots \mathrm{O} 1^{\mathrm{i}}$ & 0.95 & 2.46 & $3.304(3)$ & 148 \\
$\mathrm{C} 28-\mathrm{H} 28 \cdots \mathrm{N} 2^{\mathrm{ii}}$ & 0.95 & 2.37 & $3.262(3)$ & 157 \\
$\mathrm{C} 34-\mathrm{H} 34 \cdots \mathrm{O} 2^{\text {iii }}$ & 0.95 & 2.54 & $3.488(3)$ & 173 \\
\hline
\end{tabular}

Symmetry codes: (i) $-x+1,-y+1,-z$; (ii) $x-1, y, z$; (iii) $-x,-y+2,-z+1$.

Data collection: CrysAlis PRO (Agilent, 2011); cell refinement: CrysAlis PRO; data reduction: CrysAlis PRO; program(s) used to solve structure: SIR2014 (Burla et al., 2015); program(s) used to refine structure: SHELXL2014 (Sheldrick, 2015); molecular graphics: ORTEP-3 for Windows (Farrugia, 2012), QMOL (Gans \& Shalloway, 2001) and DIAMOND (Brandenburg, 2006); software used to prepare material for publication: MarvinSketch (ChemAxon, 2010) and publCIF (Westrip, 2010).

\section{Acknowledgements}

The Brazilian agencies CNPq (305626/2013-2 to JZ-S), CAPES, FAPESC and FAPESP (2010/10855-5 to LRSC) are acknowledged for financial support. 
Supporting information for this paper is available from the $\mathrm{IUCr}$ electronic archives (Reference: HG5431).

\section{References}

Agilent (2011). CrysAlis PRO. Agilent Technologies, Yarnton, England. Brandenburg, K. (2006). DIAMOND. Crystal Impact GbR, Bonn, Germany.
Burla, M. C., Caliandro, R., Carrozzini, B., Cascarano, G. L., Cuocci, C., Giacovazzo, C., Mallamo, M., Mazzone, A. \& Polidori, G. (2015). J. Appl. Cryst. 48, 306-309.

ChemAxon (2010). Marvinsketch. http://www.chemaxon.com.

Deobald, A. M., Camargo, L. R. S., Hörner, M., Rodrigues, O. E. D., Alves, D. \& Braga, A. L. (2011). Synthesis, pp. 2397-2406.

Farrugia, L. J. (2012). J. Appl. Cryst. 45, 849-854.

Gans, J. \& Shalloway, D. (2001). J. Mol. Graph. Model. 19, 557-559.

Pati, P. B. \& Zade, S. S. (2014). Cryst. Growth Des. 14, 1695-1700.

Sheldrick, G. M. (2015). Acta Cryst. C71, 3-8.

Westrip, S. P. (2010). J. Appl. Cryst. 43, 920-925. 


\section{supporting information}

Acta Cryst. (2015). E71, o200-o201［doi:10.1107/S2056989015003242]

\section{Crystal structure of 1-\{1-[2-(phenylselanyl)phenyl]-1 H-1,2,3-triazol-4-yl\}cyclo- hexan-1-ol}

\section{Leandro R. S. Camargo, Julio Zukerman-Schpector, Anna M. Deobald, Antonio L. Braga and Edward R. T. Tiekink}

\section{S1. Experimental}

The compound was prepared in accord with the literature (Deobald et al., 2011). Crystals were obtained by slow evaporation at room temperature from its methanol/dicloromethane $(1: 1 \mathrm{v} / \mathrm{v})$ solution.

\section{S2. Refinement}

Carbon-bound $\mathrm{H}$-atoms were placed in calculated positions $(\mathrm{C}-\mathrm{H}=0.95$ to $0.99 \AA)$ and were included in the refinement in the riding model approximation, with $U_{i s o}(\mathrm{H})=1.25 U_{e q}(\mathrm{C})$. In the same way the O-bound $\mathrm{H}$-atoms were constrained with $\mathrm{O}-\mathrm{H}=0.84 \AA$, with $U_{\text {iso }}(\mathrm{H})=1.5 U_{e q}(\mathrm{O})$. The maximum and minimum residual electron density peaks of 1.06 and 1.11 e $\AA^{-3}$, respectively were located $0.98 \AA$ and $0.84 \AA$ from the $\mathrm{Se} 2$ and Se1 atoms, respectively.

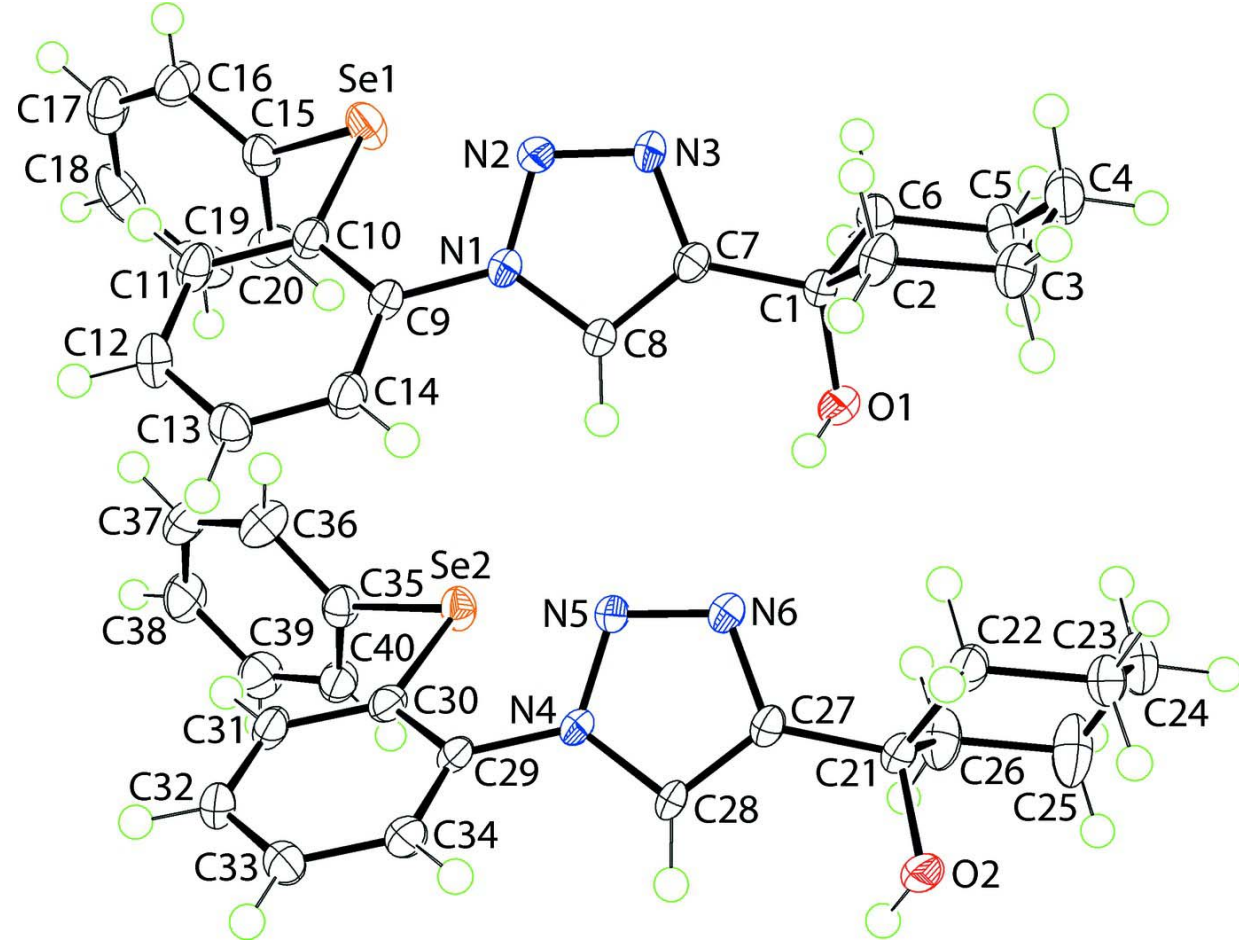

\section{Figure 1}

The molecular structures of the two independent molecules comprising the title compound showing the atom-labelling scheme and displacement ellipsoids at the $70 \%$ probability level. 


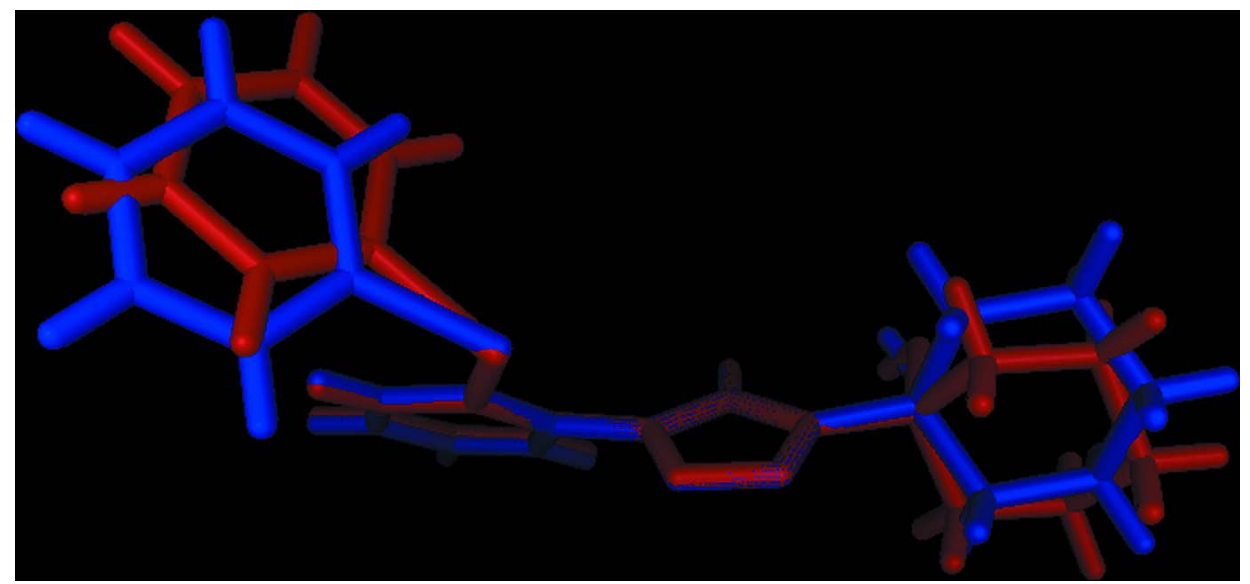

\section{Figure 2}

Superimposition of the two independent molecules. Molecule $A$ is shown in red and $B$ in blue. The molecules have been superimposed such that the triazol-4-yl rings are overlapped.

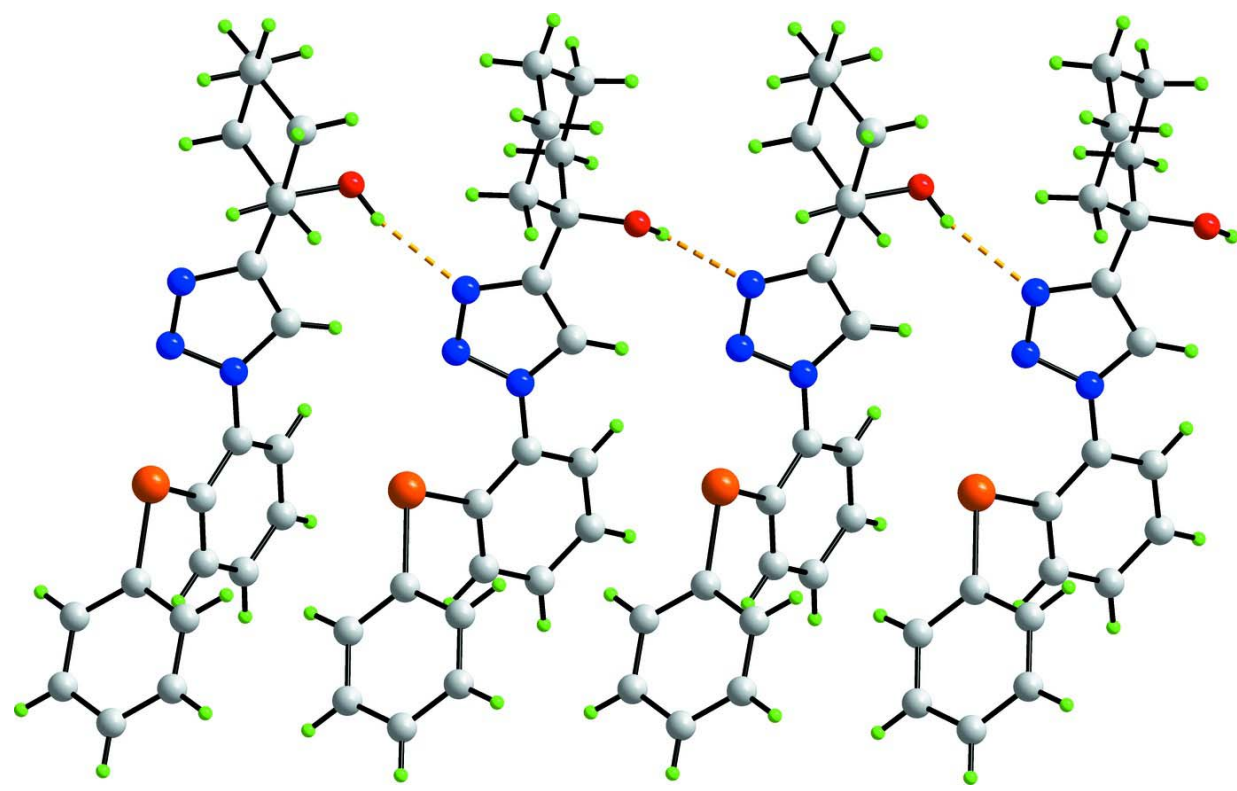

\section{Figure 3}

A view of the linear supramolecular sustained by $\mathrm{O}-\mathrm{H} \cdots \mathrm{N}$ hydrogen bonds (orange dashed lines) and aligned along the $a$ axis in the crystal packing. 


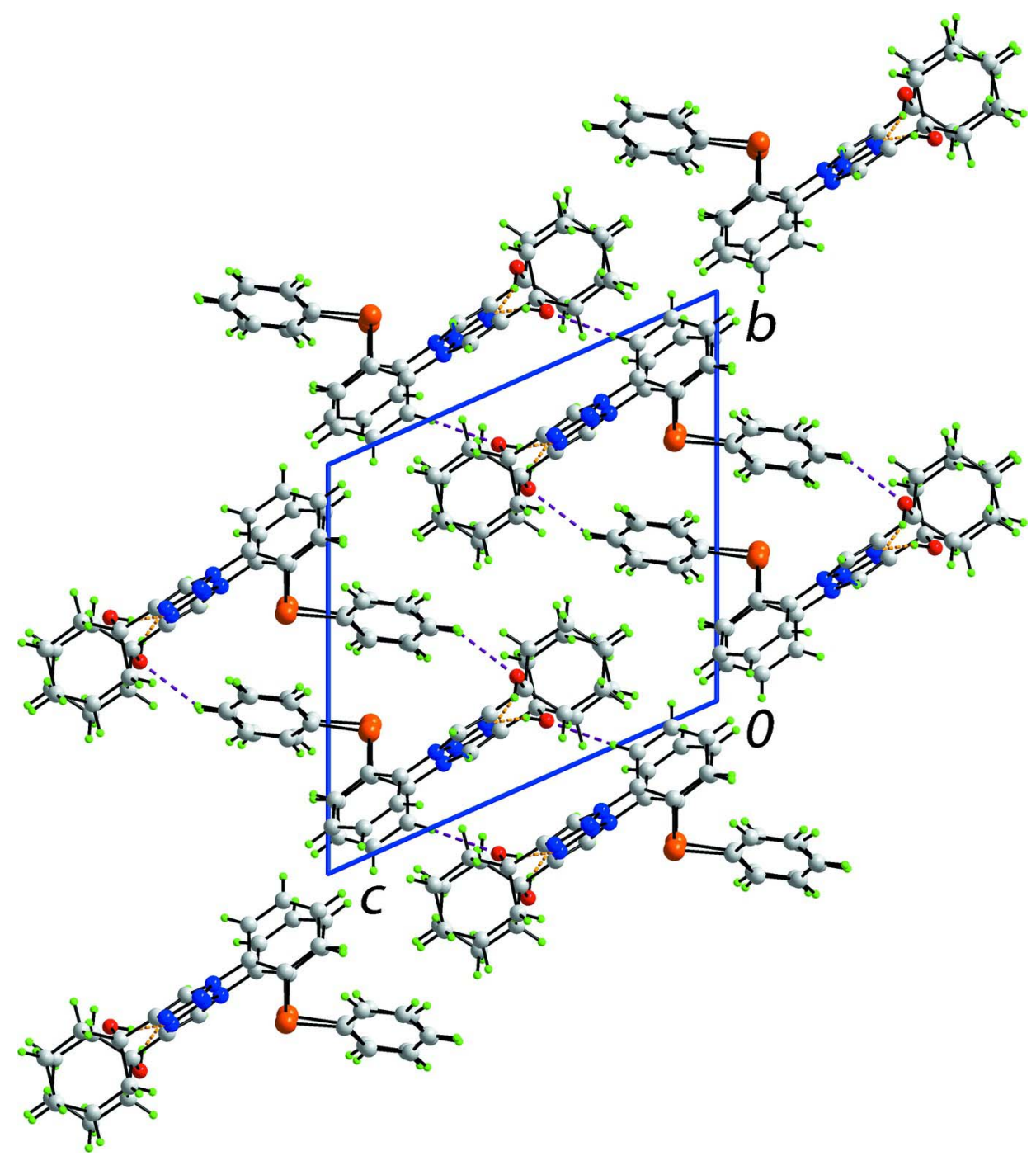

\section{Figure 4}

A view in projection down the $a$ axis of the unit-cell contents. The $\mathrm{O}-\mathrm{H} \cdots \mathrm{N}, \mathrm{C}-\mathrm{H} \cdots \mathrm{O}$ and $\mathrm{C}-\mathrm{H} \cdots \mathrm{N}$ interactions are shown as orange, purple and blue dashed lines, respectively.

\section{1-\{1-[2-(Phenylselanyl)phenyl]-1H-1,2,3-triazol-4-yl\}cyclohexan-1-ol}

\section{Crystal data}

$\mathrm{C}_{20} \mathrm{H}_{21} \mathrm{~N}_{3} \mathrm{OSe}$

$M_{r}=398.36$

Triclinic, $P \overline{1}$

$a=10.7480(4) \AA$

$b=13.7497(6) \AA$

$c=13.8849(5) \AA$

$\alpha=112.432(4)^{\circ}$

$\beta=92.889(3)^{\circ}$

$\gamma=104.059(3)^{\circ}$

$V=1816.28(13) \AA^{3}$
$Z=4$

$F(000)=816$

$D_{\mathrm{x}}=1.457 \mathrm{Mg} \mathrm{m}^{-3}$

$\mathrm{Cu} K \alpha$ radiation, $\lambda=1.54184 \AA$

Cell parameters from 19807 reflections

$\theta=3.5-74.3^{\circ}$

$\mu=2.89 \mathrm{~mm}^{-1}$

$T=100 \mathrm{~K}$

Prism, colourless

$0.20 \times 0.10 \times 0.05 \mathrm{~mm}$ 


\section{Data collection \\ Agilent SuperNova CCD diffractometer \\ Radiation source: SuperNova $(\mathrm{Cu}) \mathrm{X}$-ray Source \\ $\omega$ scans \\ Absorption correction: multi-scan \\ (CrysAlis PRO; Agilent, 2011) \\ $T_{\min }=0.884, T_{\max }=1.000$}

Refinement

Refinement on $F^{2}$

Least-squares matrix: full

$R\left[F^{2}>2 \sigma\left(F^{2}\right)\right]=0.039$

$w R\left(F^{2}\right)=0.107$

$S=1.08$

7291 reflections

453 parameters

0 restraints
26101 measured reflections

7291 independent reflections

7014 reflections with $I>2 \sigma(I)$

$R_{\text {int }}=0.087$

$\theta_{\max }=74.5^{\circ}, \theta_{\min }=3.5^{\circ}$

$h=-13 \rightarrow 13$

$k=-17 \rightarrow 17$

$l=-17 \rightarrow 16$

Hydrogen site location: inferred from neighbouring sites

$\mathrm{H}$-atom parameters constrained

$w=1 /\left[\sigma^{2}\left(F_{\mathrm{o}}^{2}\right)+(0.0551 P)^{2}+1.3888 P\right]$

where $P=\left(F_{\mathrm{o}}^{2}+2 F_{\mathrm{c}}^{2}\right) / 3$

$(\Delta / \sigma)_{\max }<0.001$

$\Delta \rho_{\max }=1.06 \mathrm{e} \AA^{-3}$

$\Delta \rho_{\min }=-1.11$ e $\AA^{-3}$

Special details

Geometry. All e.s.d.'s (except the e.s.d. in the dihedral angle between two 1.s. planes) are estimated using the full covariance matrix. The cell e.s.d.'s are taken into account individually in the estimation of e.s.d.'s in distances, angles and torsion angles; correlations between e.s.d.'s in cell parameters are only used when they are defined by crystal symmetry. An approximate (isotropic) treatment of cell e.s.d.'s is used for estimating e.s.d.'s involving 1.s. planes.

Fractional atomic coordinates and isotropic or equivalent isotropic displacement parameters $\left(\hat{A}^{2}\right)$

\begin{tabular}{|c|c|c|c|c|}
\hline & $x$ & $y$ & $z$ & $U_{\text {iso }} * / U_{\text {eq }}$ \\
\hline Se1 & $0.79205(2)$ & $0.69748(2)$ & $0.10496(2)$ & $0.01986(9)$ \\
\hline $\mathrm{O} 1$ & $0.42441(13)$ & $0.72340(12)$ & $0.48557(12)$ & $0.0183(3)$ \\
\hline $\mathrm{H} 1 \mathrm{O}$ & 0.3939 & 0.7718 & 0.4792 & $0.027 *$ \\
\hline N1 & $0.64154(16)$ & $0.81972(13)$ & $0.27686(13)$ & 0.0133 \\
\hline $\mathrm{N} 2$ & $0.75895(16)$ & $0.82424(14)$ & $0.32115(14)$ & $0.0161(4)$ \\
\hline N3 & $0.74173(17)$ & $0.80356(15)$ & $0.40452(14)$ & 0.0155 \\
\hline $\mathrm{C} 1$ & $0.56313(18)$ & $0.76209(16)$ & $0.50571(15)$ & $0.0133(4)$ \\
\hline $\mathrm{C} 2$ & $0.6108(2)$ & $0.86650(17)$ & $0.60809(16)$ & $0.0177(4)$ \\
\hline $\mathrm{H} 2 \mathrm{~A}$ & 0.7062 & 0.8956 & 0.6162 & $0.021 *$ \\
\hline $\mathrm{H} 2 \mathrm{~B}$ & 0.5715 & 0.9228 & 0.6030 & $0.021^{*}$ \\
\hline $\mathrm{C} 3$ & $0.5763(2)$ & $0.84618(18)$ & $0.70586(17)$ & $0.0205(4)$ \\
\hline $\mathrm{H} 3 \mathrm{~A}$ & 0.6154 & 0.9142 & 0.7700 & $0.025 *$ \\
\hline H3B & 0.4808 & 0.8272 & 0.7028 & $0.025^{*}$ \\
\hline $\mathrm{C} 4$ & $0.6255(2)$ & $0.75324(18)$ & $0.71257(18)$ & $0.0228(5)$ \\
\hline $\mathrm{H} 4 \mathrm{~A}$ & 0.5977 & 0.7390 & 0.7740 & $0.027^{*}$ \\
\hline H4B & 0.7217 & 0.7753 & 0.7229 & $0.027^{*}$ \\
\hline $\mathrm{C} 5$ & $0.5723(2)$ & $0.64827(18)$ & $0.61151(17)$ & $0.0207(4)$ \\
\hline $\mathrm{H} 5 \mathrm{~A}$ & 0.6074 & 0.5897 & 0.6163 & $0.025 *$ \\
\hline H5B & 0.4764 & 0.6231 & 0.6040 & $0.025 *$ \\
\hline C6 & $0.6101(2)$ & $0.66891(16)$ & $0.51505(16)$ & $0.0163(4)$ \\
\hline H6A & 0.5722 & 0.6009 & 0.4506 & $0.020 *$ \\
\hline H6B & 0.7058 & 0.6876 & 0.5199 & $0.020 *$ \\
\hline
\end{tabular}




\begin{tabular}{|c|c|c|c|c|}
\hline $\mathrm{C} 7$ & $0.61347(19)$ & $0.78607(15)$ & $0.41545(15)$ & $0.0131(4)$ \\
\hline $\mathrm{C} 8$ & $0.54837(18)$ & $0.79664(15)$ & $0.33409(15)$ & $0.0131(4)$ \\
\hline H8 & 0.4583 & 0.7895 & 0.3207 & $0.016^{*}$ \\
\hline C9 & $0.62903(18)$ & $0.84138(16)$ & $0.18508(16)$ & $0.0135(4)$ \\
\hline $\mathrm{C} 10$ & $0.69676(19)$ & 0.79898 (16) & $0.10285(16)$ & $0.0143(4)$ \\
\hline $\mathrm{C} 11$ & $0.6872(2)$ & $0.82657(17)$ & $0.01671(17)$ & $0.0178(4)$ \\
\hline H11 & 0.7361 & 0.8019 & -0.0382 & $0.021^{*}$ \\
\hline $\mathrm{C} 12$ & $0.6071(2)$ & $0.88957(17)$ & $0.01020(17)$ & $0.0189(4)$ \\
\hline H12 & 0.6005 & 0.9071 & -0.0494 & $0.023^{*}$ \\
\hline C13 & $0.5359(2)$ & $0.92755(17)$ & $0.09105(17)$ & $0.0190(4)$ \\
\hline H13 & 0.4792 & 0.9692 & 0.0856 & $0.023^{*}$ \\
\hline $\mathrm{C} 14$ & $0.5482(2)$ & $0.90447(16)$ & $0.17880(16)$ & $0.0169(4)$ \\
\hline H14 & 0.5016 & 0.9316 & 0.2347 & $0.020^{*}$ \\
\hline $\mathrm{C} 15$ & $0.7598(2)$ & $0.61407(16)$ & $-0.04505(17)$ & $0.0164(4)$ \\
\hline $\mathrm{C} 16$ & $0.8578(2)$ & $0.62498(18)$ & $-0.10508(18)$ & $0.0215(4)$ \\
\hline H16 & 0.9411 & 0.6749 & -0.0721 & $0.026^{*}$ \\
\hline $\mathrm{C} 17$ & $0.8337(3)$ & $0.5628(2)$ & $-0.2132(2)$ & $0.0277(5)$ \\
\hline H17 & 0.9011 & 0.5702 & -0.2538 & $0.033^{*}$ \\
\hline $\mathrm{C} 18$ & $0.7131(3)$ & 0.49049 (19) & $-0.26226(19)$ & $0.0286(5)$ \\
\hline H18 & 0.6973 & 0.4479 & -0.3363 & $0.034^{*}$ \\
\hline C19 & $0.6151(2)$ & $0.48054(18)$ & $-0.2025(2)$ & $0.0290(6)$ \\
\hline H19 & 0.5314 & 0.4317 & -0.2360 & $0.035^{*}$ \\
\hline $\mathrm{C} 20$ & $0.6382(2)$ & $0.54102(18)$ & -0.09454 (19) & $0.0230(5)$ \\
\hline $\mathrm{H} 20$ & 0.5708 & 0.5327 & -0.0541 & $0.028^{*}$ \\
\hline $\mathrm{Se} 2$ & $0.23728(2)$ & $0.68144(2)$ & $0.10894(2)$ & $0.01583(8)$ \\
\hline $\mathrm{O} 2$ & $-0.02727(14)$ & $0.86348(14)$ & $0.56157(12)$ & $0.0220(3)$ \\
\hline $\mathrm{H} 2 \mathrm{O}$ & -0.0859 & 0.8335 & 0.5085 & $0.033^{*}$ \\
\hline N4 & $0.15650(16)$ & $0.85609(13)$ & $0.29668(13)$ & $0.0135(3)$ \\
\hline N5 & $0.26503(16)$ & $0.83739(15)$ & $0.33016(14)$ & $0.0169(4)$ \\
\hline N6 & $0.24879(17)$ & $0.82511(15)$ & $0.41776(15)$ & $0.0172(4)$ \\
\hline $\mathrm{C} 21$ & $0.08158(19)$ & $0.82042(17)$ & $0.53679(16)$ & $0.0155(4)$ \\
\hline $\mathrm{C} 22$ & $0.1860(2)$ & 0.88455 (17) & $0.63518(16)$ & $0.0171(4)$ \\
\hline $\mathrm{H} 22 \mathrm{~A}$ & 0.2685 & 0.8672 & 0.6183 & $0.021^{*}$ \\
\hline $\mathrm{H} 22 \mathrm{~B}$ & 0.2007 & 0.9641 & 0.6550 & $0.021^{*}$ \\
\hline $\mathrm{C} 23$ & $0.1488(2)$ & $0.85807(18)$ & $0.72899(17)$ & $0.0204(4)$ \\
\hline $\mathrm{H} 23 \mathrm{~A}$ & 0.2214 & 0.8980 & 0.7889 & $0.025^{*}$ \\
\hline H23B & 0.0720 & 0.8833 & 0.7514 & $0.025^{*}$ \\
\hline $\mathrm{C} 24$ & $0.1178(2)$ & $0.7356(2)$ & $0.70098(19)$ & $0.0267(5)$ \\
\hline $\mathrm{H} 24 \mathrm{~A}$ & 0.0915 & 0.7205 & 0.7624 & $0.032 *$ \\
\hline H24B & 0.1963 & 0.7111 & 0.6835 & $0.032 *$ \\
\hline $\mathrm{C} 25$ & $0.0081(3)$ & $0.6720(2)$ & $0.60657(18)$ & $0.0300(6)$ \\
\hline $\mathrm{H} 25 \mathrm{~A}$ & -0.0093 & 0.5923 & 0.5876 & $0.036^{*}$ \\
\hline $\mathrm{H} 25 \mathrm{~B}$ & -0.0722 & 0.6926 & 0.6259 & $0.036^{*}$ \\
\hline $\mathrm{C} 26$ & $0.0444(2)$ & $0.69690(18)$ & $0.51152(17)$ & $0.0233(5)$ \\
\hline $\mathrm{H} 26 \mathrm{~A}$ & -0.0301 & 0.6583 & 0.4531 & $0.028^{*}$ \\
\hline $\mathrm{H} 26 \mathrm{~B}$ & 0.1184 & 0.6682 & 0.4873 & $0.028^{*}$ \\
\hline $\mathrm{C} 27$ & $0.13023(19)$ & $0.83756(16)$ & $0.44306(15)$ & $0.0142(4)$ \\
\hline $\mathrm{C} 28$ & $0.07058(19)$ & $0.85768(16)$ & $0.36567(15)$ & $0.0146(4)$ \\
\hline
\end{tabular}




$\begin{array}{lllll}\text { H28 } & -0.0123 & 0.8700 & 0.3612 & 0.017^{*} \\ \text { C29 } & 0.14779(18) & 0.87469(16) & 0.20299(16) & 0.0137(4) \\ \text { C30 } & 0.18517(18) & 0.80731(16) & 0.11135(16) & 0.0140(4) \\ \text { C31 } & 0.18131(19) & 0.83302(17) & 0.02395(17) & 0.0165(4) \\ \text { H31 } & 0.2096 & 0.7903 & -0.0379 & 0.020^{*} \\ \text { C32 } & 0.1367(2) & 0.92036(17) & 0.02572(17) & 0.0177(4) \\ \text { H32 } & 0.1346 & 0.9369 & -0.0347 & 0.021^{*} \\ \text { C33 } & 0.0950(2) & 0.98361(17) & 0.11573(17) & 0.0185(4) \\ \text { H33 } & 0.0626 & 1.0421 & 0.1161 & 0.022^{*} \\ \text { C34 } & 0.10072(19) & 0.96140(17) & 0.20476(16) & 0.0163(4) \\ \text { H34 } & 0.0729 & 1.0047 & 0.2665 & 0.020^{*} \\ \text { C35 } & 0.2304(2) & 0.60755(16) & -0.04120(17) & 0.0161(4) \\ \text { C36 } & 0.3411(2) & 0.62954(18) & -0.08591(19) & 0.0230(5) \\ \text { H36 } & 0.4187 & 0.6821 & -0.0428 & 0.028^{*} \\ \text { C37 } & 0.3381(2) & 0.5748(2) & -0.19308(19) & 0.0251(5) \\ \text { H37 } & 0.4135 & 0.5906 & -0.2235 & 0.030^{*} \\ \text { C38 } & 0.2256(2) & 0.49665(19) & -0.25669(18) & 0.0241(5) \\ \text { H38 } & 0.2239 & 0.4594 & -0.3303 & 0.029^{*} \\ \text { C39 } & 0.1154(2) & 0.47333(18) & -0.21178(18) & 0.0223(5) \\ \text { H39 } & 0.0386 & 0.4195 & -0.2548 & 0.027^{*} \\ \text { C40 } & 0.1176(2) & 0.52865(17) & -0.10406(17) & 0.0181(4) \\ \text { H40 } & 0.0423 & 0.5126 & -0.0735 & 0.022^{*}\end{array}$

Atomic displacement parameters $\left(\AA^{2}\right)$

\begin{tabular}{lllllll}
\hline & $U^{11}$ & $U^{22}$ & $U^{33}$ & $U^{12}$ & $U^{13}$ & $U^{33}$ \\
\hline $\mathrm{Se} 1$ & $0.02619(14)$ & $0.02673(14)$ & $0.01324(14)$ & $0.01777(10)$ & $0.00654(10)$ & $0.00845(10)$ \\
$\mathrm{O} 1$ & $0.0129(7)$ & $0.0239(7)$ & $0.0216(8)$ & $0.0041(6)$ & $0.0051(6)$ & $0.0134(6)$ \\
$\mathrm{N} 1$ & $0.0133(8)$ & $0.0166(8)$ & $0.0119(8)$ & $0.0052(6)$ & $0.0047(6)$ & $0.0069(6)$ \\
$\mathrm{N} 2$ & $0.0119(8)$ & $0.0250(9)$ & $0.0134(9)$ & $0.0061(7)$ & $0.0033(6)$ & $0.0092(7)$ \\
$\mathrm{N} 3$ & $0.0154(8)$ & $0.0227(8)$ & $0.0124(8)$ & $0.0073(7)$ & $0.0060(7)$ & $0.0099(7)$ \\
$\mathrm{C} 1$ & $0.0118(9)$ & $0.0176(9)$ & $0.0112(9)$ & $0.0034(7)$ & $0.0028(7)$ & $0.0070(8)$ \\
$\mathrm{C} 2$ & $0.0222(10)$ & $0.0174(9)$ & $0.0135(10)$ & $0.0061(8)$ & $0.0079(8)$ & $0.0056(8)$ \\
$\mathrm{C} 3$ & $0.0247(10)$ & $0.0231(10)$ & $0.0137(10)$ & $0.0074(8)$ & $0.0067(8)$ & $0.0067(8)$ \\
$\mathrm{C} 4$ & $0.0268(11)$ & $0.0272(11)$ & $0.0161(11)$ & $0.0057(9)$ & $0.0043(9)$ & $0.0116(9)$ \\
$\mathrm{C} 5$ & $0.0261(11)$ & $0.0217(10)$ & $0.0180(11)$ & $0.0060(8)$ & $0.0039(9)$ & $0.0125(9)$ \\
$\mathrm{C} 6$ & $0.0203(10)$ & $0.0167(9)$ & $0.0146(10)$ & $0.0072(8)$ & $0.0049(8)$ & $0.0077(8)$ \\
$\mathrm{C} 7$ & $0.0141(9)$ & $0.0132(8)$ & $0.0115(9)$ & $0.0044(7)$ & $0.0042(7)$ & $0.0040(7)$ \\
$\mathrm{C} 8$ & $0.0125(8)$ & $0.0167(9)$ & $0.0120(9)$ & $0.0044(7)$ & $0.0040(7)$ & $0.0075(7)$ \\
$\mathrm{C} 9$ & $0.0137(9)$ & $0.0145(8)$ & $0.0137(10)$ & $0.0034(7)$ & $0.0046(7)$ & $0.0072(7)$ \\
$\mathrm{C} 10$ & $0.0154(9)$ & $0.0147(9)$ & $0.0154(10)$ & $0.0056(7)$ & $0.0062(8)$ & $0.0078(7)$ \\
$\mathrm{C} 11$ & $0.0217(10)$ & $0.0199(10)$ & $0.0150(10)$ & $0.0080(8)$ & $0.0099(8)$ & $0.0083(8)$ \\
$\mathrm{C} 12$ & $0.0266(11)$ & $0.0186(9)$ & $0.0142(10)$ & $0.0065(8)$ & $0.0053(8)$ & $0.0091(8)$ \\
$\mathrm{C} 13$ & $0.0240(10)$ & $0.0193(9)$ & $0.0201(11)$ & $0.0121(8)$ & $0.0066(9)$ & $0.0109(8)$ \\
$\mathrm{C} 14$ & $0.0195(10)$ & $0.0189(9)$ & $0.0152(10)$ & $0.0077(8)$ & $0.0070(8)$ & $0.0083(8)$ \\
$\mathrm{C} 15$ & $0.0202(10)$ & $0.0164(9)$ & $0.0163(10)$ & $0.0087(8)$ & $0.0047(8)$ & $0.0082(8)$ \\
C16 & $0.0198(10)$ & $0.0245(10)$ & $0.0198(11)$ & $0.0067(8)$ & $0.0079(9)$ & $0.0078(9)$ \\
C17 & $0.0369(13)$ & $0.0317(12)$ & $0.0225(12)$ & $0.0165(10)$ & $0.0149(10)$ & $0.0142(10)$
\end{tabular}




$\begin{array}{lllllll}\text { C18 } & 0.0481(15) & 0.0245(11) & 0.0135(11) & 0.0198(11) & -0.0015(10) & 0.0030(9) \\ \text { C19 } & 0.0266(12) & 0.0167(10) & 0.0354(14) & 0.0042(9) & -0.0078(10) & 0.0046(10) \\ \text { C20 } & 0.0185(10) & 0.0212(10) & 0.0304(13) & 0.0056(8) & 0.0072(9) & 0.0114(9) \\ \text { Se2 } & 0.02069(13) & 0.01657(13) & 0.01253(14) & 0.00764(9) & 0.00494(9) & 0.00664(9) \\ \text { O2 } & 0.0158(7) & 0.0399(9) & 0.0138(7) & 0.0139(6) & 0.0063(6) & 0.0106(6) \\ \text { N4 } & 0.0128(7) & 0.0188(8) & 0.0128(8) & 0.0072(6) & 0.0065(6) & 0.0082(7) \\ \text { N5 } & 0.0136(8) & 0.0296(9) & 0.0116(8) & 0.0088(7) & 0.0044(7) & 0.0108(7) \\ \text { N6 } & 0.0157(8) & 0.0265(9) & 0.0144(9) & 0.0100(7) & 0.0071(7) & 0.0107(7) \\ \text { C21 } & 0.0138(9) & 0.0221(10) & 0.0121(9) & 0.0062(8) & 0.0054(7) & 0.0075(8) \\ \text { C22 } & 0.0174(9) & 0.0208(10) & 0.0131(10) & 0.0049(8) & 0.0049(8) & 0.0068(8) \\ \text { C23 } & 0.0222(10) & 0.0280(11) & 0.0116(10) & 0.0085(9) & 0.0040(8) & 0.0077(8) \\ \text { C24 } & 0.0339(13) & 0.0313(12) & 0.0201(12) & 0.0079(10) & 0.0066(10) & 0.0167(10) \\ \text { C25 } & 0.0413(14) & 0.0263(11) & 0.0189(12) & -0.0009(10) & 0.0073(11) & 0.0117(9) \\ \text { C26 } & 0.0294(11) & 0.0206(10) & 0.0144(10) & -0.0005(9) & 0.0036(9) & 0.0058(8) \\ \text { C27 } & 0.0148(9) & 0.0177(9) & 0.0112(9) & 0.0055(7) & 0.0055(7) & 0.0060(7) \\ \text { C28 } & 0.0136(9) & 0.0219(9) & 0.0115(9) & 0.0068(7) & 0.0069(7) & 0.0088(8) \\ \text { C29 } & 0.0101(8) & 0.0186(9) & 0.0138(10) & 0.0033(7) & 0.0047(7) & 0.0080(8) \\ \text { C30 } & 0.0109(8) & 0.0159(9) & 0.0139(10) & 0.0022(7) & 0.0029(7) & 0.0058(8) \\ \text { C31 } & 0.0158(9) & 0.0208(10) & 0.0143(10) & 0.0048(8) & 0.0065(8) & 0.0085(8) \\ \text { C32 } & 0.0181(10) & 0.0236(10) & 0.0148(10) & 0.0056(8) & 0.0047(8) & 0.0114(8) \\ \text { C33 } & 0.0200(10) & 0.0240(10) & 0.0176(10) & 0.0112(8) & 0.0054(8) & 0.0115(8) \\ \text { C34 } & 0.0160(9) & 0.0203(9) & 0.0146(10) & 0.0080(8) & 0.0054(8) & 0.0070(8) \\ \text { C35 } & 0.0199(10) & 0.0151(9) & 0.0151(10) & 0.0072(8) & 0.0058(8) & 0.0065(8) \\ \text { C36 } & 0.0206(10) & 0.0231(10) & 0.0240(12) & 0.0057(8) & 0.0096(9) & 0.0078(9) \\ \text { C37 } & 0.0221(11) & 0.0336(12) & 0.0230(12) & 0.0103(9) & 0.0127(9) & 0.0123(10) \\ \text { C38 } & 0.0309(12) & 0.0294(11) & 0.0133(10) & 0.0136(10) & 0.0085(9) & 0.0063(9) \\ \text { C39 } & 0.0242(11) & 0.0222(10) & 0.0184(11) & 0.0066(9) & 0.0037(9) & 0.0061(9) \\ \text { C40 } & 0.0180(10) & 0.0199(10) & 0.0166(10) & 0.0046(8) & 0.0046(8) & 0.0080(8)\end{array}$

Geometric parameters $\left(\AA,{ }^{\circ}\right)$

\begin{tabular}{llll}
\hline $\mathrm{Se} 1-\mathrm{C} 15$ & $1.920(2)$ & $\mathrm{Se} 2-\mathrm{C} 35$ & $1.927(2)$ \\
$\mathrm{Se} 1-\mathrm{C} 10$ & $1.929(2)$ & $\mathrm{Se} 2-\mathrm{C} 30$ & $1.934(2)$ \\
$\mathrm{O} 1-\mathrm{C} 1$ & $1.429(2)$ & $\mathrm{O} 2-\mathrm{C} 21$ & 0.8400 \\
$\mathrm{O} 1-\mathrm{H} 1 \mathrm{O}$ & 0.8400 & $\mathrm{O} 2-\mathrm{H} 2 \mathrm{O}$ & $1.351(2)$ \\
$\mathrm{N} 1-\mathrm{N} 2$ & $1.352(2)$ & $\mathrm{N} 4-\mathrm{N} 5$ & $1.361(2)$ \\
$\mathrm{N} 1-\mathrm{C} 8$ & $1.361(2)$ & $\mathrm{N} 4-\mathrm{C} 28$ & $1.421(3)$ \\
$\mathrm{N} 1-\mathrm{C} 9$ & $1.422(3)$ & $\mathrm{N} 4-\mathrm{C} 29$ & $1.305(3)$ \\
$\mathrm{N} 2-\mathrm{N} 3$ & $1.305(3)$ & $\mathrm{N} 5-\mathrm{N} 6$ & $1.372(3)$ \\
$\mathrm{N} 3-\mathrm{C} 7$ & $1.367(3)$ & $\mathrm{N} 6-\mathrm{C} 27$ & $1.505(3)$ \\
$\mathrm{C} 1-\mathrm{C} 7$ & $1.506(3)$ & $\mathrm{C} 21-\mathrm{C} 27$ & $1.533(3)$ \\
$\mathrm{C} 1-\mathrm{C} 6$ & $1.532(3)$ & $\mathrm{C} 21-\mathrm{C} 22$ & $1.539(3)$ \\
$\mathrm{C} 1-\mathrm{C} 2$ & $1.535(3)$ & $\mathrm{C} 21-\mathrm{C} 26$ & $1.527(3)$ \\
$\mathrm{C} 2-\mathrm{C} 3$ & $1.532(3)$ & $\mathrm{C} 22-\mathrm{C} 23$ & 0.9900 \\
$\mathrm{C} 2-\mathrm{H} 2 \mathrm{~A}$ & 0.9900 & $\mathrm{C} 22-\mathrm{H} 22 \mathrm{~A}$ & 0.9900 \\
$\mathrm{C} 2-\mathrm{H} 2 \mathrm{~B}$ & 0.9900 & $\mathrm{C} 22-\mathrm{H} 22 \mathrm{~B}$ & $1.521(3)$ \\
$\mathrm{C} 3-\mathrm{C} 4$ & $1.528(3)$ & $\mathrm{C} 23-\mathrm{C} 24$ & 0.9900 \\
$\mathrm{C} 3-\mathrm{H} 3 \mathrm{~A}$ & 0.9900 & $\mathrm{C} 23-\mathrm{H} 23 \mathrm{~A}$ &
\end{tabular}




\begin{tabular}{|c|c|c|c|}
\hline $\mathrm{C} 3-\mathrm{H} 3 \mathrm{~B}$ & 0.9900 & $\mathrm{C} 23-\mathrm{H} 23 \mathrm{~B}$ & 0.9900 \\
\hline $\mathrm{C} 4-\mathrm{C} 5$ & $1.534(3)$ & $\mathrm{C} 24-\mathrm{C} 25$ & $1.531(3)$ \\
\hline $\mathrm{C} 4-\mathrm{H} 4 \mathrm{~A}$ & 0.9900 & $\mathrm{C} 24-\mathrm{H} 24 \mathrm{~A}$ & 0.9900 \\
\hline $\mathrm{C} 4-\mathrm{H} 4 \mathrm{~B}$ & 0.9900 & $\mathrm{C} 24-\mathrm{H} 24 \mathrm{~B}$ & 0.9900 \\
\hline $\mathrm{C} 5-\mathrm{C} 6$ & $1.526(3)$ & $\mathrm{C} 25-\mathrm{C} 26$ & $1.531(3)$ \\
\hline $\mathrm{C} 5-\mathrm{H} 5 \mathrm{~A}$ & 0.9900 & $\mathrm{C} 25-\mathrm{H} 25 \mathrm{~A}$ & 0.9900 \\
\hline $\mathrm{C} 5-\mathrm{H} 5 \mathrm{~B}$ & 0.9900 & $\mathrm{C} 25-\mathrm{H} 25 \mathrm{~B}$ & 0.9900 \\
\hline C6-H6A & 0.9900 & $\mathrm{C} 26-\mathrm{H} 26 \mathrm{~A}$ & 0.9900 \\
\hline $\mathrm{C} 6-\mathrm{H} 6 \mathrm{~B}$ & 0.9900 & $\mathrm{C} 26-\mathrm{H} 26 \mathrm{~B}$ & 0.9900 \\
\hline $\mathrm{C} 7-\mathrm{C} 8$ & $1.372(3)$ & $\mathrm{C} 27-\mathrm{C} 28$ & $1.373(3)$ \\
\hline $\mathrm{C} 8-\mathrm{H} 8$ & 0.9500 & $\mathrm{C} 28-\mathrm{H} 28$ & 0.9500 \\
\hline $\mathrm{C} 9-\mathrm{C} 14$ & $1.390(3)$ & $\mathrm{C} 29-\mathrm{C} 34$ & $1.396(3)$ \\
\hline $\mathrm{C} 9-\mathrm{C} 10$ & $1.398(3)$ & $\mathrm{C} 29-\mathrm{C} 30$ & $1.404(3)$ \\
\hline $\mathrm{C} 10-\mathrm{C} 11$ & $1.392(3)$ & $\mathrm{C} 30-\mathrm{C} 31$ & $1.389(3)$ \\
\hline $\mathrm{C} 11-\mathrm{C} 12$ & $1.383(3)$ & $\mathrm{C} 31-\mathrm{C} 32$ & $1.390(3)$ \\
\hline C11-H11 & 0.9500 & $\mathrm{C} 31-\mathrm{H} 31$ & 0.9500 \\
\hline $\mathrm{C} 12-\mathrm{C} 13$ & $1.397(3)$ & $\mathrm{C} 32-\mathrm{C} 33$ & $1.391(3)$ \\
\hline $\mathrm{C} 12-\mathrm{H} 12$ & 0.9500 & $\mathrm{C} 32-\mathrm{H} 32$ & 0.9500 \\
\hline $\mathrm{C} 13-\mathrm{C} 14$ & $1.379(3)$ & $\mathrm{C} 33-\mathrm{C} 34$ & $1.384(3)$ \\
\hline C13-H13 & 0.9500 & $\mathrm{C} 33-\mathrm{H} 33$ & 0.9500 \\
\hline C14-H14 & 0.9500 & C34-H34 & 0.9500 \\
\hline $\mathrm{C} 15-\mathrm{C} 20$ & $1.391(3)$ & $\mathrm{C} 35-\mathrm{C} 36$ & $1.392(3)$ \\
\hline $\mathrm{C} 15-\mathrm{C} 16$ & $1.391(3)$ & $\mathrm{C} 35-\mathrm{C} 40$ & $1.393(3)$ \\
\hline $\mathrm{C} 16-\mathrm{C} 17$ & $1.387(3)$ & $\mathrm{C} 36-\mathrm{C} 37$ & $1.382(3)$ \\
\hline $\mathrm{C} 16-\mathrm{H} 16$ & 0.9500 & $\mathrm{C} 36-\mathrm{H} 36$ & 0.9500 \\
\hline $\mathrm{C} 17-\mathrm{C} 18$ & $1.379(4)$ & $\mathrm{C} 37-\mathrm{C} 38$ & $1.390(3)$ \\
\hline C17-H17 & 0.9500 & C37-H37 & 0.9500 \\
\hline $\mathrm{C} 18-\mathrm{C} 19$ & $1.386(4)$ & $\mathrm{C} 38-\mathrm{C} 39$ & $1.391(3)$ \\
\hline C18-H18 & 0.9500 & $\mathrm{C} 38-\mathrm{H} 38$ & 0.9500 \\
\hline $\mathrm{C} 19-\mathrm{C} 20$ & $1.382(4)$ & $\mathrm{C} 39-\mathrm{C} 40$ & $1.391(3)$ \\
\hline C19-H19 & 0.9500 & C39-H39 & 0.9500 \\
\hline $\mathrm{C} 20-\mathrm{H} 20$ & 0.9500 & $\mathrm{C} 40-\mathrm{H} 40$ & 0.9500 \\
\hline $\mathrm{C} 15-\mathrm{Se} 1-\mathrm{C} 10$ & $95.64(9)$ & $\mathrm{C} 35-\mathrm{Se} 2-\mathrm{C} 30$ & $98.68(9)$ \\
\hline $\mathrm{C} 1-\mathrm{O} 1-\mathrm{H} 1 \mathrm{O}$ & 109.5 & $\mathrm{C} 21-\mathrm{O} 2-\mathrm{H} 2 \mathrm{O}$ & 109.5 \\
\hline $\mathrm{N} 2-\mathrm{N} 1-\mathrm{C} 8$ & $110.56(16)$ & $\mathrm{N} 5-\mathrm{N} 4-\mathrm{C} 28$ & $110.57(17)$ \\
\hline $\mathrm{N} 2-\mathrm{N} 1-\mathrm{C} 9$ & $120.58(16)$ & $\mathrm{N} 5-\mathrm{N} 4-\mathrm{C} 29$ & $119.73(16)$ \\
\hline $\mathrm{C} 8-\mathrm{N} 1-\mathrm{C} 9$ & $128.83(17)$ & $\mathrm{C} 28-\mathrm{N} 4-\mathrm{C} 29$ & $129.65(17)$ \\
\hline $\mathrm{N} 3-\mathrm{N} 2-\mathrm{N} 1$ & $107.20(16)$ & $\mathrm{N} 6-\mathrm{N} 5-\mathrm{N} 4$ & $107.29(16)$ \\
\hline $\mathrm{N} 2-\mathrm{N} 3-\mathrm{C} 7$ & $109.58(17)$ & $\mathrm{N} 5-\mathrm{N} 6-\mathrm{C} 27$ & $109.59(17)$ \\
\hline $\mathrm{O} 1-\mathrm{C} 1-\mathrm{C} 7$ & $110.33(16)$ & $\mathrm{O} 2-\mathrm{C} 21-\mathrm{C} 27$ & $110.34(17)$ \\
\hline $\mathrm{O} 1-\mathrm{C} 1-\mathrm{C} 6$ & $105.90(16)$ & $\mathrm{O} 2-\mathrm{C} 21-\mathrm{C} 22$ & $105.70(16)$ \\
\hline $\mathrm{C} 7-\mathrm{C} 1-\mathrm{C} 6$ & $109.86(16)$ & $\mathrm{C} 27-\mathrm{C} 21-\mathrm{C} 22$ & $110.48(16)$ \\
\hline $\mathrm{O} 1-\mathrm{C} 1-\mathrm{C} 2$ & $110.90(16)$ & $\mathrm{O} 2-\mathrm{C} 21-\mathrm{C} 26$ & $111.10(17)$ \\
\hline $\mathrm{C} 7-\mathrm{C} 1-\mathrm{C} 2$ & $109.18(16)$ & $\mathrm{C} 27-\mathrm{C} 21-\mathrm{C} 26$ & $108.70(16)$ \\
\hline $\mathrm{C} 6-\mathrm{C} 1-\mathrm{C} 2$ & $110.64(17)$ & $\mathrm{C} 22-\mathrm{C} 21-\mathrm{C} 26$ & $110.50(18)$ \\
\hline $\mathrm{C} 3-\mathrm{C} 2-\mathrm{C} 1$ & $112.56(17)$ & $\mathrm{C} 23-\mathrm{C} 22-\mathrm{C} 21$ & $112.61(17)$ \\
\hline $\mathrm{C} 3-\mathrm{C} 2-\mathrm{H} 2 \mathrm{~A}$ & 109.1 & $\mathrm{C} 23-\mathrm{C} 22-\mathrm{H} 22 \mathrm{~A}$ & 109.1 \\
\hline
\end{tabular}




\begin{tabular}{|c|c|c|c|}
\hline $\mathrm{C} 1-\mathrm{C} 2-\mathrm{H} 2 \mathrm{~A}$ & 109.1 & $\mathrm{C} 21-\mathrm{C} 22-\mathrm{H} 22 \mathrm{~A}$ & 109.1 \\
\hline $\mathrm{C} 3-\mathrm{C} 2-\mathrm{H} 2 \mathrm{~B}$ & 109.1 & $\mathrm{C} 23-\mathrm{C} 22-\mathrm{H} 22 \mathrm{~B}$ & 109.1 \\
\hline $\mathrm{C} 1-\mathrm{C} 2-\mathrm{H} 2 \mathrm{~B}$ & 109.1 & $\mathrm{C} 21-\mathrm{C} 22-\mathrm{H} 22 \mathrm{~B}$ & 109.1 \\
\hline $\mathrm{H} 2 \mathrm{~A}-\mathrm{C} 2-\mathrm{H} 2 \mathrm{~B}$ & 107.8 & $\mathrm{H} 22 \mathrm{~A}-\mathrm{C} 22-\mathrm{H} 22 \mathrm{~B}$ & 107.8 \\
\hline $\mathrm{C} 4-\mathrm{C} 3-\mathrm{C} 2$ & $111.17(17)$ & $\mathrm{C} 24-\mathrm{C} 23-\mathrm{C} 22$ & $111.29(17)$ \\
\hline $\mathrm{C} 4-\mathrm{C} 3-\mathrm{H} 3 \mathrm{~A}$ & 109.4 & $\mathrm{C} 24-\mathrm{C} 23-\mathrm{H} 23 \mathrm{~A}$ & 109.4 \\
\hline $\mathrm{C} 2-\mathrm{C} 3-\mathrm{H} 3 \mathrm{~A}$ & 109.4 & $\mathrm{C} 22-\mathrm{C} 23-\mathrm{H} 23 \mathrm{~A}$ & 109.4 \\
\hline $\mathrm{C} 4-\mathrm{C} 3-\mathrm{H} 3 \mathrm{~B}$ & 109.4 & $\mathrm{C} 24-\mathrm{C} 23-\mathrm{H} 23 \mathrm{~B}$ & 109.4 \\
\hline $\mathrm{C} 2-\mathrm{C} 3-\mathrm{H} 3 \mathrm{~B}$ & 109.4 & $\mathrm{C} 22-\mathrm{C} 23-\mathrm{H} 23 \mathrm{~B}$ & 109.4 \\
\hline $\mathrm{H} 3 \mathrm{~A}-\mathrm{C} 3-\mathrm{H} 3 \mathrm{~B}$ & 108.0 & $\mathrm{H} 23 \mathrm{~A}-\mathrm{C} 23-\mathrm{H} 23 \mathrm{~B}$ & 108.0 \\
\hline $\mathrm{C} 3-\mathrm{C} 4-\mathrm{C} 5$ & $110.64(19)$ & $\mathrm{C} 23-\mathrm{C} 24-\mathrm{C} 25$ & $110.2(2)$ \\
\hline $\mathrm{C} 3-\mathrm{C} 4-\mathrm{H} 4 \mathrm{~A}$ & 109.5 & $\mathrm{C} 23-\mathrm{C} 24-\mathrm{H} 24 \mathrm{~A}$ & 109.6 \\
\hline $\mathrm{C} 5-\mathrm{C} 4-\mathrm{H} 4 \mathrm{~A}$ & 109.5 & $\mathrm{C} 25-\mathrm{C} 24-\mathrm{H} 24 \mathrm{~A}$ & 109.6 \\
\hline $\mathrm{C} 3-\mathrm{C} 4-\mathrm{H} 4 \mathrm{~B}$ & 109.5 & $\mathrm{C} 23-\mathrm{C} 24-\mathrm{H} 24 \mathrm{~B}$ & 109.6 \\
\hline $\mathrm{C} 5-\mathrm{C} 4-\mathrm{H} 4 \mathrm{~B}$ & 109.5 & $\mathrm{C} 25-\mathrm{C} 24-\mathrm{H} 24 \mathrm{~B}$ & 109.6 \\
\hline $\mathrm{H} 4 \mathrm{~A}-\mathrm{C} 4-\mathrm{H} 4 \mathrm{~B}$ & 108.1 & $\mathrm{H} 24 \mathrm{~A}-\mathrm{C} 24-\mathrm{H} 24 \mathrm{~B}$ & 108.1 \\
\hline $\mathrm{C} 6-\mathrm{C} 5-\mathrm{C} 4$ & $110.60(18)$ & $\mathrm{C} 26-\mathrm{C} 25-\mathrm{C} 24$ & $110.7(2)$ \\
\hline $\mathrm{C} 6-\mathrm{C} 5-\mathrm{H} 5 \mathrm{~A}$ & 109.5 & $\mathrm{C} 26-\mathrm{C} 25-\mathrm{H} 25 \mathrm{~A}$ & 109.5 \\
\hline $\mathrm{C} 4-\mathrm{C} 5-\mathrm{H} 5 \mathrm{~A}$ & 109.5 & $\mathrm{C} 24-\mathrm{C} 25-\mathrm{H} 25 \mathrm{~A}$ & 109.5 \\
\hline $\mathrm{C} 6-\mathrm{C} 5-\mathrm{H} 5 \mathrm{~B}$ & 109.5 & $\mathrm{C} 26-\mathrm{C} 25-\mathrm{H} 25 \mathrm{~B}$ & 109.5 \\
\hline $\mathrm{C} 4-\mathrm{C} 5-\mathrm{H} 5 \mathrm{~B}$ & 109.5 & $\mathrm{C} 24-\mathrm{C} 25-\mathrm{H} 25 \mathrm{~B}$ & 109.5 \\
\hline $\mathrm{H} 5 \mathrm{~A}-\mathrm{C} 5-\mathrm{H} 5 \mathrm{~B}$ & 108.1 & $\mathrm{H} 25 \mathrm{~A}-\mathrm{C} 25-\mathrm{H} 25 \mathrm{~B}$ & 108.1 \\
\hline $\mathrm{C} 5-\mathrm{C} 6-\mathrm{C} 1$ & $112.04(16)$ & $\mathrm{C} 25-\mathrm{C} 26-\mathrm{C} 21$ & $112.70(18)$ \\
\hline $\mathrm{C} 5-\mathrm{C} 6-\mathrm{H} 6 \mathrm{~A}$ & 109.2 & $\mathrm{C} 25-\mathrm{C} 26-\mathrm{H} 26 \mathrm{~A}$ & 109.1 \\
\hline $\mathrm{C} 1-\mathrm{C} 6-\mathrm{H} 6 \mathrm{~A}$ & 109.2 & $\mathrm{C} 21-\mathrm{C} 26-\mathrm{H} 26 \mathrm{~A}$ & 109.1 \\
\hline $\mathrm{C} 5-\mathrm{C} 6-\mathrm{H} 6 \mathrm{~B}$ & 109.2 & $\mathrm{C} 25-\mathrm{C} 26-\mathrm{H} 26 \mathrm{~B}$ & 109.1 \\
\hline $\mathrm{C} 1-\mathrm{C} 6-\mathrm{H} 6 \mathrm{~B}$ & 109.2 & $\mathrm{C} 21-\mathrm{C} 26-\mathrm{H} 26 \mathrm{~B}$ & 109.1 \\
\hline $\mathrm{H} 6 \mathrm{~A}-\mathrm{C} 6-\mathrm{H} 6 \mathrm{~B}$ & 107.9 & $\mathrm{H} 26 \mathrm{~A}-\mathrm{C} 26-\mathrm{H} 26 \mathrm{~B}$ & 107.8 \\
\hline $\mathrm{N} 3-\mathrm{C} 7-\mathrm{C} 8$ & $108.03(18)$ & $\mathrm{N} 6-\mathrm{C} 27-\mathrm{C} 28$ & $107.76(17)$ \\
\hline $\mathrm{N} 3-\mathrm{C} 7-\mathrm{C} 1$ & $122.24(18)$ & $\mathrm{N} 6-\mathrm{C} 27-\mathrm{C} 21$ & $121.25(18)$ \\
\hline $\mathrm{C} 8-\mathrm{C} 7-\mathrm{C} 1$ & $129.71(18)$ & $\mathrm{C} 28-\mathrm{C} 27-\mathrm{C} 21$ & $130.79(18)$ \\
\hline $\mathrm{N} 1-\mathrm{C} 8-\mathrm{C} 7$ & $104.62(17)$ & $\mathrm{N} 4-\mathrm{C} 28-\mathrm{C} 27$ & $104.77(17)$ \\
\hline $\mathrm{N} 1-\mathrm{C} 8-\mathrm{H} 8$ & 127.7 & $\mathrm{~N} 4-\mathrm{C} 28-\mathrm{H} 28$ & 127.6 \\
\hline $\mathrm{C} 7-\mathrm{C} 8-\mathrm{H} 8$ & 127.7 & $\mathrm{C} 27-\mathrm{C} 28-\mathrm{H} 28$ & 127.6 \\
\hline $\mathrm{C} 14-\mathrm{C} 9-\mathrm{C} 10$ & $121.08(19)$ & $\mathrm{C} 34-\mathrm{C} 29-\mathrm{C} 30$ & $121.17(19)$ \\
\hline $\mathrm{C} 14-\mathrm{C} 9-\mathrm{N} 1$ & $118.83(17)$ & $\mathrm{C} 34-\mathrm{C} 29-\mathrm{N} 4$ & $118.11(17)$ \\
\hline $\mathrm{C} 10-\mathrm{C} 9-\mathrm{N} 1$ & $120.09(18)$ & $\mathrm{C} 30-\mathrm{C} 29-\mathrm{N} 4$ & $120.71(18)$ \\
\hline $\mathrm{C} 11-\mathrm{C} 10-\mathrm{C} 9$ & $118.36(19)$ & $\mathrm{C} 31-\mathrm{C} 30-\mathrm{C} 29$ & $118.17(19)$ \\
\hline $\mathrm{C} 11-\mathrm{C} 10-\mathrm{Se} 1$ & $120.90(15)$ & $\mathrm{C} 31-\mathrm{C} 30-\mathrm{Se} 2$ & $121.99(15)$ \\
\hline $\mathrm{C} 9-\mathrm{C} 10-\mathrm{Se} 1$ & $120.62(16)$ & $\mathrm{C} 29-\mathrm{C} 30-\mathrm{Se} 2$ & $119.83(16)$ \\
\hline $\mathrm{C} 12-\mathrm{C} 11-\mathrm{C} 10$ & $120.68(19)$ & $\mathrm{C} 30-\mathrm{C} 31-\mathrm{C} 32$ & $120.90(19)$ \\
\hline $\mathrm{C} 12-\mathrm{C} 11-\mathrm{H} 11$ & 119.7 & $\mathrm{C} 30-\mathrm{C} 31-\mathrm{H} 31$ & 119.5 \\
\hline $\mathrm{C} 10-\mathrm{C} 11-\mathrm{H} 11$ & 119.7 & $\mathrm{C} 32-\mathrm{C} 31-\mathrm{H} 31$ & 119.5 \\
\hline $\mathrm{C} 11-\mathrm{C} 12-\mathrm{C} 13$ & $120.2(2)$ & $\mathrm{C} 31-\mathrm{C} 32-\mathrm{C} 33$ & $120.2(2)$ \\
\hline $\mathrm{C} 11-\mathrm{C} 12-\mathrm{H} 12$ & 119.9 & $\mathrm{C} 31-\mathrm{C} 32-\mathrm{H} 32$ & 119.9 \\
\hline $\mathrm{C} 13-\mathrm{C} 12-\mathrm{H} 12$ & 119.9 & $\mathrm{C} 33-\mathrm{C} 32-\mathrm{H} 32$ & 119.9 \\
\hline $\mathrm{C} 14-\mathrm{C} 13-\mathrm{C} 12$ & $119.8(2)$ & $\mathrm{C} 34-\mathrm{C} 33-\mathrm{C} 32$ & $120.1(2)$ \\
\hline $\mathrm{C} 14-\mathrm{C} 13-\mathrm{H} 13$ & 120.1 & $\mathrm{C} 34-\mathrm{C} 33-\mathrm{H} 33$ & 120.0 \\
\hline
\end{tabular}




\begin{tabular}{|c|c|c|c|}
\hline $\mathrm{C} 12-\mathrm{C} 13-\mathrm{H} 13$ & 120.1 & $\mathrm{C} 32-\mathrm{C} 33-\mathrm{H} 33$ & 120.0 \\
\hline $\mathrm{C} 13-\mathrm{C} 14-\mathrm{C} 9$ & $119.76(19)$ & $\mathrm{C} 33-\mathrm{C} 34-\mathrm{C} 29$ & $119.39(18)$ \\
\hline $\mathrm{C} 13-\mathrm{C} 14-\mathrm{H} 14$ & 120.1 & $\mathrm{C} 33-\mathrm{C} 34-\mathrm{H} 34$ & 120.3 \\
\hline $\mathrm{C} 9-\mathrm{C} 14-\mathrm{H} 14$ & 120.1 & $\mathrm{C} 29-\mathrm{C} 34-\mathrm{H} 34$ & 120.3 \\
\hline $\mathrm{C} 20-\mathrm{C} 15-\mathrm{C} 16$ & $119.4(2)$ & $\mathrm{C} 36-\mathrm{C} 35-\mathrm{C} 40$ & $119.97(19)$ \\
\hline $\mathrm{C} 20-\mathrm{C} 15-\mathrm{Se} 1$ & $120.11(16)$ & $\mathrm{C} 36-\mathrm{C} 35-\mathrm{Se} 2$ & $119.49(16)$ \\
\hline $\mathrm{C} 16-\mathrm{C} 15-\mathrm{Se} 1$ & $120.51(16)$ & $\mathrm{C} 40-\mathrm{C} 35-\mathrm{Se} 2$ & $120.46(15)$ \\
\hline $\mathrm{C} 17-\mathrm{C} 16-\mathrm{C} 15$ & $119.9(2)$ & $\mathrm{C} 37-\mathrm{C} 36-\mathrm{C} 35$ & $119.9(2)$ \\
\hline $\mathrm{C} 17-\mathrm{C} 16-\mathrm{H} 16$ & 120.1 & $\mathrm{C} 37-\mathrm{C} 36-\mathrm{H} 36$ & 120.1 \\
\hline $\mathrm{C} 15-\mathrm{C} 16-\mathrm{H} 16$ & 120.1 & $\mathrm{C} 35-\mathrm{C} 36-\mathrm{H} 36$ & 120.1 \\
\hline $\mathrm{C} 18-\mathrm{C} 17-\mathrm{C} 16$ & $120.7(2)$ & $\mathrm{C} 36-\mathrm{C} 37-\mathrm{C} 38$ & $120.6(2)$ \\
\hline $\mathrm{C} 18-\mathrm{C} 17-\mathrm{H} 17$ & 119.6 & $\mathrm{C} 36-\mathrm{C} 37-\mathrm{H} 37$ & 119.7 \\
\hline $\mathrm{C} 16-\mathrm{C} 17-\mathrm{H} 17$ & 119.6 & $\mathrm{C} 38-\mathrm{C} 37-\mathrm{H} 37$ & 119.7 \\
\hline $\mathrm{C} 17-\mathrm{C} 18-\mathrm{C} 19$ & $119.3(2)$ & $\mathrm{C} 37-\mathrm{C} 38-\mathrm{C} 39$ & $119.6(2)$ \\
\hline $\mathrm{C} 17-\mathrm{C} 18-\mathrm{H} 18$ & 120.3 & $\mathrm{C} 37-\mathrm{C} 38-\mathrm{H} 38$ & 120.2 \\
\hline $\mathrm{C} 19-\mathrm{C} 18-\mathrm{H} 18$ & 120.3 & $\mathrm{C} 39-\mathrm{C} 38-\mathrm{H} 38$ & 120.2 \\
\hline $\mathrm{C} 20-\mathrm{C} 19-\mathrm{C} 18$ & $120.6(2)$ & $\mathrm{C} 38-\mathrm{C} 39-\mathrm{C} 40$ & $120.1(2)$ \\
\hline $\mathrm{C} 20-\mathrm{C} 19-\mathrm{H} 19$ & 119.7 & $\mathrm{C} 38-\mathrm{C} 39-\mathrm{H} 39$ & 119.9 \\
\hline $\mathrm{C} 18-\mathrm{C} 19-\mathrm{H} 19$ & 119.7 & $\mathrm{C} 40-\mathrm{C} 39-\mathrm{H} 39$ & 119.9 \\
\hline $\mathrm{C} 19-\mathrm{C} 20-\mathrm{C} 15$ & $120.1(2)$ & $\mathrm{C} 39-\mathrm{C} 40-\mathrm{C} 35$ & $119.8(2)$ \\
\hline $\mathrm{C} 19-\mathrm{C} 20-\mathrm{H} 20$ & 119.9 & $\mathrm{C} 39-\mathrm{C} 40-\mathrm{H} 40$ & 120.1 \\
\hline $\mathrm{C} 15-\mathrm{C} 20-\mathrm{H} 20$ & 119.9 & $\mathrm{C} 35-\mathrm{C} 40-\mathrm{H} 40$ & 120.1 \\
\hline $\mathrm{C} 8-\mathrm{N} 1-\mathrm{N} 2-\mathrm{N} 3$ & $0.6(2)$ & $\mathrm{C} 28-\mathrm{N} 4-\mathrm{N} 5-\mathrm{N} 6$ & $1.1(2)$ \\
\hline $\mathrm{C} 9-\mathrm{N} 1-\mathrm{N} 2-\mathrm{N} 3$ & $178.54(16)$ & $\mathrm{C} 29-\mathrm{N} 4-\mathrm{N} 5-\mathrm{N} 6$ & $178.85(17)$ \\
\hline $\mathrm{N} 1-\mathrm{N} 2-\mathrm{N} 3-\mathrm{C} 7$ & $-0.3(2)$ & $\mathrm{N} 4-\mathrm{N} 5-\mathrm{N} 6-\mathrm{C} 27$ & $-1.0(2)$ \\
\hline $\mathrm{O} 1-\mathrm{C} 1-\mathrm{C} 2-\mathrm{C} 3$ & $64.5(2)$ & $\mathrm{O} 2-\mathrm{C} 21-\mathrm{C} 22-\mathrm{C} 23$ & $68.3(2)$ \\
\hline $\mathrm{C} 7-\mathrm{C} 1-\mathrm{C} 2-\mathrm{C} 3$ & $-173.77(17)$ & $\mathrm{C} 27-\mathrm{C} 21-\mathrm{C} 22-\mathrm{C} 23$ & $-172.32(17)$ \\
\hline $\mathrm{C} 6-\mathrm{C} 1-\mathrm{C} 2-\mathrm{C} 3$ & $-52.7(2)$ & $\mathrm{C} 26-\mathrm{C} 21-\mathrm{C} 22-\mathrm{C} 23$ & $-52.0(2)$ \\
\hline $\mathrm{C} 1-\mathrm{C} 2-\mathrm{C} 3-\mathrm{C} 4$ & $54.3(2)$ & $\mathrm{C} 21-\mathrm{C} 22-\mathrm{C} 23-\mathrm{C} 24$ & $55.7(2)$ \\
\hline $\mathrm{C} 2-\mathrm{C} 3-\mathrm{C} 4-\mathrm{C} 5$ & $-56.0(2)$ & $\mathrm{C} 22-\mathrm{C} 23-\mathrm{C} 24-\mathrm{C} 25$ & $-57.6(2)$ \\
\hline $\mathrm{C} 3-\mathrm{C} 4-\mathrm{C} 5-\mathrm{C} 6$ & $57.4(2)$ & $\mathrm{C} 23-\mathrm{C} 24-\mathrm{C} 25-\mathrm{C} 26$ & $57.4(3)$ \\
\hline $\mathrm{C} 4-\mathrm{C} 5-\mathrm{C} 6-\mathrm{C} 1$ & $-56.9(2)$ & $\mathrm{C} 24-\mathrm{C} 25-\mathrm{C} 26-\mathrm{C} 21$ & $-55.4(3)$ \\
\hline $\mathrm{O} 1-\mathrm{C} 1-\mathrm{C} 6-\mathrm{C} 5$ & $-66.1(2)$ & $\mathrm{O} 2-\mathrm{C} 21-\mathrm{C} 26-\mathrm{C} 25$ & $-64.8(2)$ \\
\hline $\mathrm{C} 7-\mathrm{C} 1-\mathrm{C} 6-\mathrm{C} 5$ & $174.77(16)$ & $\mathrm{C} 27-\mathrm{C} 21-\mathrm{C} 26-\mathrm{C} 25$ & $173.55(19)$ \\
\hline $\mathrm{C} 2-\mathrm{C} 1-\mathrm{C} 6-\mathrm{C} 5$ & $54.2(2)$ & $\mathrm{C} 22-\mathrm{C} 21-\mathrm{C} 26-\mathrm{C} 25$ & $52.2(2)$ \\
\hline $\mathrm{N} 2-\mathrm{N} 3-\mathrm{C} 7-\mathrm{C} 8$ & $0.0(2)$ & $\mathrm{N} 5-\mathrm{N} 6-\mathrm{C} 27-\mathrm{C} 28$ & $0.5(2)$ \\
\hline $\mathrm{N} 2-\mathrm{N} 3-\mathrm{C} 7-\mathrm{C} 1$ & $-178.91(17)$ & $\mathrm{N} 5-\mathrm{N} 6-\mathrm{C} 27-\mathrm{C} 21$ & $175.98(18)$ \\
\hline $\mathrm{O} 1-\mathrm{C} 1-\mathrm{C} 7-\mathrm{N} 3$ & $-167.04(17)$ & $\mathrm{O} 2-\mathrm{C} 21-\mathrm{C} 27-\mathrm{N} 6$ & $165.99(17)$ \\
\hline $\mathrm{C} 6-\mathrm{C} 1-\mathrm{C} 7-\mathrm{N} 3$ & $-50.6(2)$ & $\mathrm{C} 22-\mathrm{C} 21-\mathrm{C} 27-\mathrm{N} 6$ & $49.5(2)$ \\
\hline $\mathrm{C} 2-\mathrm{C} 1-\mathrm{C} 7-\mathrm{N} 3$ & $70.8(2)$ & $\mathrm{C} 26-\mathrm{C} 21-\mathrm{C} 27-\mathrm{N} 6$ & $-71.9(2)$ \\
\hline $\mathrm{O} 1-\mathrm{C} 1-\mathrm{C} 7-\mathrm{C} 8$ & $14.3(3)$ & $\mathrm{O} 2-\mathrm{C} 21-\mathrm{C} 27-\mathrm{C} 28$ & $-19.7(3)$ \\
\hline $\mathrm{C} 6-\mathrm{C} 1-\mathrm{C} 7-\mathrm{C} 8$ & $130.7(2)$ & $\mathrm{C} 22-\mathrm{C} 21-\mathrm{C} 27-\mathrm{C} 28$ & $-136.3(2)$ \\
\hline $\mathrm{C} 2-\mathrm{C} 1-\mathrm{C} 7-\mathrm{C} 8$ & $-107.8(2)$ & $\mathrm{C} 26-\mathrm{C} 21-\mathrm{C} 27-\mathrm{C} 28$ & $102.3(2)$ \\
\hline $\mathrm{N} 2-\mathrm{N} 1-\mathrm{C} 8-\mathrm{C} 7$ & $-0.5(2)$ & N5-N4-C28-C27 & $-0.8(2)$ \\
\hline $\mathrm{C} 9-\mathrm{N} 1-\mathrm{C} 8-\mathrm{C} 7$ & $-178.31(17)$ & $\mathrm{C} 29-\mathrm{N} 4-\mathrm{C} 28-\mathrm{C} 27$ & $-178.21(18)$ \\
\hline $\mathrm{N} 3-\mathrm{C} 7-\mathrm{C} 8-\mathrm{N} 1$ & $0.3(2)$ & $\mathrm{N} 6-\mathrm{C} 27-\mathrm{C} 28-\mathrm{N} 4$ & $0.2(2)$ \\
\hline $\mathrm{C} 1-\mathrm{C} 7-\mathrm{C} 8-\mathrm{N} 1$ & $179.13(18)$ & $\mathrm{C} 21-\mathrm{C} 27-\mathrm{C} 28-\mathrm{N} 4$ & $-174.70(19)$ \\
\hline
\end{tabular}




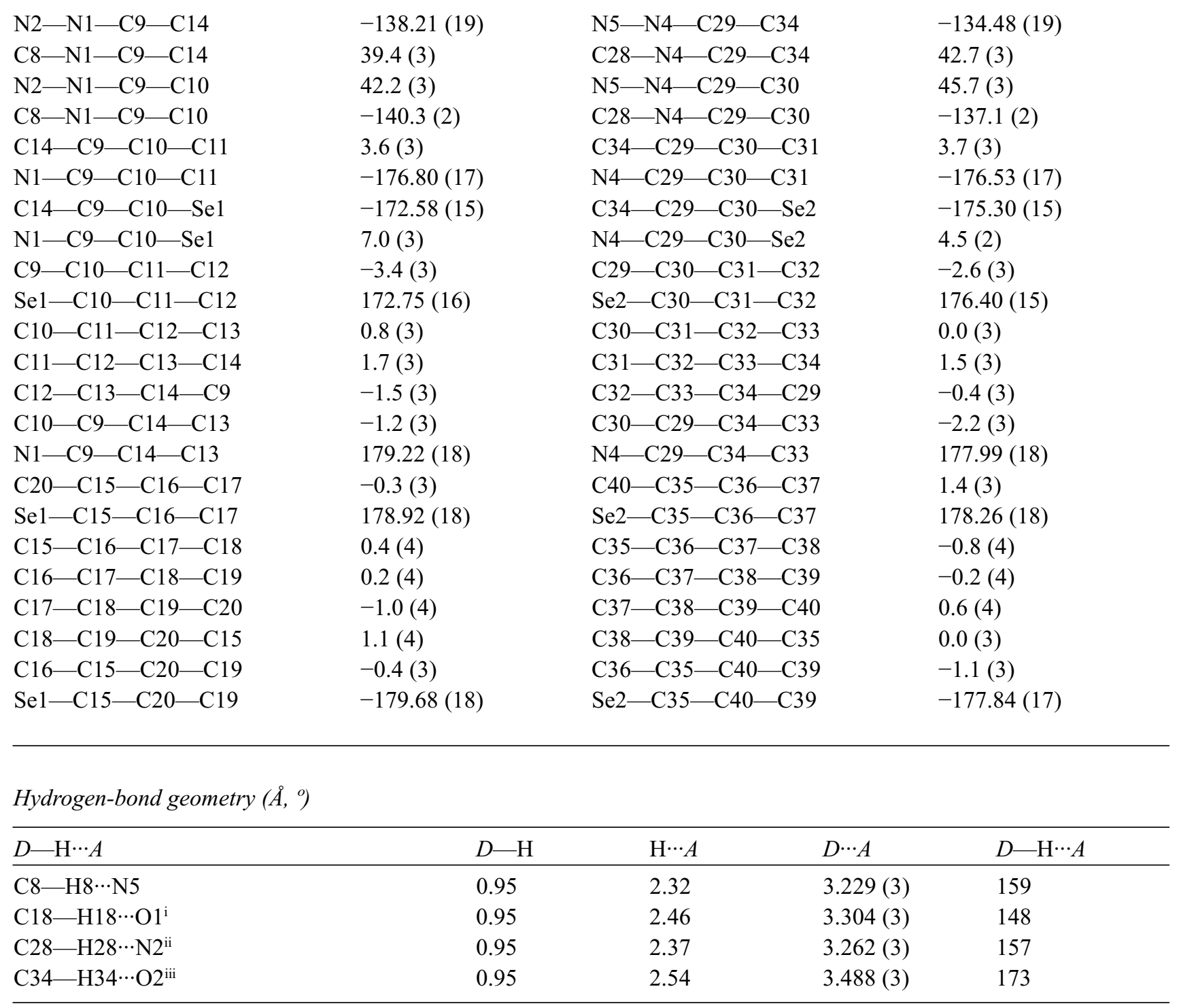

Symmetry codes: (i) $-x+1,-y+1,-z$; (ii) $x-1, y, z$; (iii) $-x,-y+2,-z+1$. 